\title{
UNUSUAL SENSORY PHENOMENA FOLLOWING REMOVAL OF A TUMOUR OF THE SENSORY CORTEX.
}

\author{
By I. M. ALLEN,* London.
}

THE case here reported presented several unusual features, the most interesting of which were the variations in the appreciation of the shape, size, form and nature of objects for a short period following the removal of a tumour from the postrolandic cerebral cortex. It is with the object of placing on record the observations made upon these phenomena that the case is published. It is necessary, however, in order to indicate the background upon which the phenomena appeared, to describe the whole case in detail.

\section{GLINICAL GASE.}

E. E., male, age 48 years, was admitted complaining of (1) difficulty in using the right arm and leg; and (2) attacks of "pins and needles" associated with movements on the right side. The symptoms had been present for fifteen months, but there had been some difficulty in moving the right foot for ten years.

History of Present Illness.-In 1918 the patient was wounded by a piece of shrapnel which passed through the back of the right thigh, and by other pieces which lodged in the inner side of the right ankle. After nine months in hospital he recovered and was discharged from the army with "slight foot-drop" on the right side. Excepting for a slight limp he was perfectly well until fifteen months before admission.

The present illness began suddenly fifteen months before admission with an attack of "pins and needles," which started in the right foot and travelled up the right leg. Following this attack, all movements of the right foot were lost for a time, but after a short interval the foot could again be moved a short distance upwards. There was no further attack for six months. The second attack occurred six months before admission. It began with "pins and needles" in the right foot; this sensation travelled up the right leg; convulsive movements appeared at the right knee; the sensation passed up the right side of the body to the neck; convulsive movements appeared at the right shoulder; the sensation then travelled to the right side of the face and down the right arm to the finger tips.

During the next nine months the attacks occurred in series at intervals of about one week. After each, the right arm and leg were useless but recovered in from five to six hours. The attacks varied in intensity ; they were often slight and produced little general disturbance; but on one occasion it was so severe that the patient fell to the floor and was unable to move for three hours. In none was consciousness lost.

Since the first attack there had been some motor disability on the right side. This appeared first in the right foot, then in the whole leg and finally also in the right arm. The right side of the face became weak shortly before admission.

Speech was sometimes affected either at the end of or after the attack. As far as the patient could remember, the speech defect appeared first when the march of the sensation

*From the West End Hospital for Nervous Diseases. London. 
had almost ceased and persisted for a few minutes after the attack had ended. During the previous three months, it had been noticed that he sometimes "stuttered" when he became excited.

Each attack was succeeded by extreme exhaustion, a feeling of depression and a sensation of " pain and tenderness on the top of the head." Headache, which usually appeared as " a sense of pressure on the top of the head but deep in," sometimes persisted for twenty-four hours and sometimes for five days. A tender area was often felt on the scalp a little to the left of the middle line.

Vision was not obviously impaired but spots sometimes appeared in front of the eyes. The sight of the left eye had been defective for many years following a blow on the eyeball.

There was a slight sensation of giddiness on turning to the right. This had been present more or less all the time since the onset of the illness, but was severe on only two occasions when, on turning to the right, equilibrium was completely lost. There had been no romiting. The organic functions were normal.

Pain had been present constantly, " deep in the right arm below the shoulder," for fifteen months, and had been very severe during the last two months. There had been frequent attacks of crying and sobbing "as though his heart would break" for half an hour at a time. Such attacks appeared frequently for two or three days in succession, during which the patient would have to lock himself away from other people. They had become much more severe and frequent during the last four months. On two occasions, the first four months before admission, the patient had become much more hilarious than was usual for him ; he could not stop laughing and had to leave the room for some time until the laughing ceased of its own accord. During the fifteen months he had always felt unusually distressed when he saw a child suffering pain and felt that he himself experienced all the pain the child suffered. Though he had never known what fear was, he had on two occasions been aware of a feeling of extreme dread for no apparent reason. On the first occasion, when he had reached a seat in the back of the circle of a theatre, he at once "felt an awful dread of falling forward into the pit below." He felt so terrified that he had to get a transfer to a seat on the ground floor, and when he reached it the fear disappeared. He had this experience four months before admission and again had the same feeling two months later in the same part of another theatre. The attacks of sobbing were much more likely to occur on what were described as "depressing days," when the patient also felt cold and shivered so much that he had to don warmer clothing. There had keen no sensations of warmth. Throughout the whole of his illness the patient had been exceedingly reticent about his emotional experiences because he was afraid that people would think he was parading a war disability.

Previous History.-Twenty-eight years before he had received a blow on the head and was not well until two weeks later. In addition to the wounds mentioned above he received a superficial shrapnel wound in the right temporal region. The family history was regative.

rondition on Examination.-His mental condition was normal. Memory, attention $\mathrm{r}$ i cooperation were perfectly good.

Cranial Nerves.-The pupils were small, equal and reacted normally. Vision was normal in the right eye, but limited to the perception of fingers in the left eye. There were no obvious changes in the visual fields. Optic discs and fundi : on the right side the fundus was normal; there was a large patch of opaque nerve fibres at the upper and outer disc margin; the physiological cup was not obliterated; the outline of the disc was blurred; the surface was apparently raised and the veins were distended and pulsating. On the left side the view of the fundus was obscured by the presence of small lenticular opacities. Ocular movements were normal; there was no nystagmus; hearing was normal and equal on the two sides. Speech : there was some hesitation over individual words; he complained that sometimes, if the idea were difficult, the words would not come though he knew what he wanted to say. Trigeminal nerve: the jaw swung a little 
to the right on opening; otherwise normal. Facial nerve : voluntary movements were weak on the right side; emotional and associated movements were equal and normal. The other cranial nerves were normal.

Sensory System.- Spontaneous abnormal sensations : sensory attacks confined to the right side as described in the history; pain deep in the right arm. Objectively, slight impairment of cotton wool touches over the right arm and leg and the right side of the body, most evident over the lower third of the leg and foot. Pain : the finer variations were impaired over the area indicated above, compared with the left side. Thermal : the finer variations were impaired in the above area. Deep pressure : normal in the right arm ; impaired in the right leg. Posture : good in the right hand and fingers; absent in the right ankle and toes. Vibration: impaired in the right arm and leg compared with the left. Localisation : impaired in the right arm; a longer period elapsed before the response than on the left side. Appreciation of size and shape : there was slight impairment over the right hand and more over the right leg. All forms of sensibility were impaired over the body and limbs on the right side as compared with the left.

Motor System.-Cpper limbs : the left arm was normal; the right arm tended to assume a position of flexion; tone was increased especially in the flexors; motor power was diminished for all movements ; supinator, biceps and triceps jerks were exaggerated and more active than those on the left side; Léri's forearm sign was present; the Mayer sign was absent ; and the Klippel-Weil sign was present.

Lower limbs : the left leg was normal ; the right leg showed a tendency to assume a position of full extension; tone was increased especially in the extensors ; motor power was diminished for all movements ; the knee-jerks and ankle-jerks were exaggerated and more active than on the left side ; patellar and ankle clonus was present ; and the plantar reflex resulted in dorsiflexion. The abdominal reflexes on the right side were less active than those on the left and they tired more rapidly.

Gait : the right leg swung in a semicircle from the hip, was thrown forward and brought to the floor with a stamp.

Spontaneous movements : clonic movements were present at intervals in the right quadriceps.

Coordination: with the finger-nose test there was a coarse tremor of the right arm and hand at the end of the movement, increased with the eyes closed; there was no Rombergism. The sphincters were normal.

Cerebrospinal fluid: not under increased pressure; clear; two small lymphocytes per c.mm.; total protein, 0.04 per cent. ; no excess of globulin; Wassermann reaction negative; Lange test-0122100000. Blood: Wassermann reaction negative.

Skull : there was a small tender area over the vertex on the left side of the middle line and about the upper limit of the fissure of Rolando. X-ray of the skull : there was a gap in the inner table about the mid-point of the vertex ; as this corresponded with the fontanelle, it was regarded as a normal finding.

The digestive, cardiac, and respiratory systems were normal. The blood-vessels were normal. Pulse : 80, regular; blood pressure-115 systolic, 80 diastolic. Vasomotor system : on two occasions the right hand swelled and became cyanosed; on each occasion the swelling disappeared during the night; the right arm and leg were definitely colder to the touch than the left.

Subsequent progress: Two attacks were observed. The first occurred during the night and lasted eighteen minutes. A tingling sensation was felt in the toes of the right foot; convulsive movements then appeared there; the sensation travelled to the right knee; movements appeared there; the sensation then travelled up the right side of the body to the right side of the face and head; movements appeared at the right shoulder; and the sensation then travelled down the right arm to the finger tips. The patient was conscious throughout the attack ; but towards the end of it he was unable to speak. The right arm and leg were flaccid for five hours afterwards. 
A second attack occurred after an interval of ten days and was observed personally. Tingling developed in the toes of the right foot and slowly travelled to the right knee; movement then commenced in the toes and spread up to the knee. Up to the middle of the body the movements followed behind the sensation but there overtook it and spread to the right shoulder. Meanwhile the sensation travelled up the right side of the body to the face, at which stage the movements at the shoulder ceased. The sensation then travelled slowly down the right arm to the finger tips. The patient was conscious throughout the whole attack; his speech was unaffected, and he could describe the position of the sensation from stage to stage. In the body the movements were bilateral and could be felt marching up the trunk part by part. In the neck, violent movements resulted from contractions of the right sternomastoid before movements of the arm commenced. Contractions of the left sternomastoid also occurred but it was not at all certain that they were not due to the general movements of the head as a result of the contractions of the muscle on the other side. Immediately after this attack, slight tingling was felt again in the toes of the right foot but did not spread elsewhere. The right arm and leg were flaccid and did not recover their normal tone and power for fortyfive hours. During that time the patient was generally depressed, and complained that whenever he attempted to use the right arm or leg he felt "a sense of pulling or pressure in the head," over the whole vertex but more obviously on the left side than on the right.

Surgical Treatment.-A diagnosis was made of a neoplasm of the postrolandic sensory cortex on the left side, with involvement chiefly of the leg area close to the middle line and of the parts adjacent to it anteriorly and laterally. The operation was performed by Mr. Cecil P. G. Wakeley. A large osteoplastic flap was turned back from the middle line on the left side and the left cerebral hemisphere in front of and behind the fissure of Rolando exposed. The membranes bulged through the wound excepting close to the middle line. On incising the dura it was found to be adherent to the underlying structures over the postrolandic cortex close to the middle line. In this situation the tissues were not displaced into the wound following the incision of the dura but elsewhere the brain tissue expanded into the wound. A large tumour was shelled out from the site of the postrolandic cortex close to the middle line. It occupied. the whole of the leg area, was slightly overlapped by the brain tissue all round and displaced the brain tissue mainly inferiorly but also laterally, anteriorly and posteriorly. The dura, osteoplastic flap and scalp were closed in the usual way.

Pathological Report on the Tumour.-The pathological report on the tumour, provided by Dr. W. E. Carnegie Dickson, was as follows. The tumour consisted of a hemispherical mass about half the size of a small to medium sized orange, two and a quarter to two and a half inches in diameter and one and a half inches thick (vide Figure). Its surface was lobulated and simulated the surface appearance of the brain in colour and appearance but was more irregular. The tumour was fairly firm and on section showed a more or less homogeneous slight yellowish-white appearance. On microscopical examination the tumour was much "whorled" but showed no psammomata or calcification. There was a considerable amount of fibrous tissue framework, and the tumourcells were not of the actively malignant type but probably mildly and locally so. The tumour was a meningioma.

P'ost-operatice Findings.-Observations commenced an hour and a half after the operation when the patient had recovered from the anæsthetic. He was then able to speak freely and could produce a sentence of five or six words with ease. Occasionally, he stressed each word with great emphasis and definitely paused between individual words. Sometimes he found difficulty in producing a word he wanted to say, and paused for an appreciable interval before he could say it. He complained of slight pain in the right leg and of a very severe pain "deep in the right arm above the elbow." There was conjugate deviation of the eyes to the left and a persistent tendency to rotate the head towards the right. The right arm and leg were completely flaccid and on the right side no tendon reflexes could be elicited. 
Five and a half hours after the operation the speech defect was more pronounced and the conjugate deviation of the eyes and the tendency to rotate the head had disappeared. Nine and a half hours after the operation the difficulty in finding words was more pronounced and more frequent. The patient would say three or four words perfectly well, then stop and indicate that he could speak no further. After a short rest he could proceed as before but soon met with the same difficulty.

Twenty-four hours after the operation his condition was as follows. The speech defect was worse. The patient complained that after saying about three words with ease, when he wanted to say the next word, several seemed to come into his mind at once and he had difficulty in choosing the right one. Following this he could not speak at all until he had a rest. He hesitated frequently and often could not complete a word he had begun. There was slight weakness of the right side of the face for voluntary movements. Upper limbs : motor power was completely absent in the right arm; there was some return of tone to the flexor muscles; and the tendon jerks were more exag-

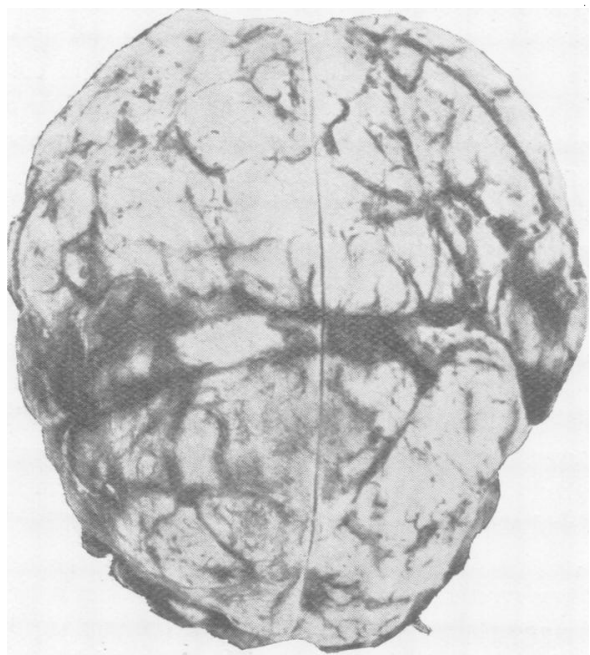

Photograph of Tumour-actual size (Dr. Carnegie Dickson).

gerated than those on the left side. The abdominal reflexes were absent on the right side. Lower limbs : motor power was poor; there was slight spasticity in the right leg; the right knee jerk was more active than the left; and the right plantar reflex resulted in dorsiflexion.

On the second day the speech condition was unchanged. Pain "deep in the right arm " was still present; but there was no pain in the leg. "Twitching movements" appeared at intervals in the right foot.

On the third day the attacks associated with movements of the right foot were continuing as before. An attack associated with movements of the right side of the face and of both upper eyelids was observed in the evening. A twitching movement appeared at the right angle of the mouth and was followed immediately by clonic blinking movements of both upper eyelids. The two movements then proceeded together until the end of the attack which often lasted as long as three or four minutes. During the attack the patient could not speak but was able to point to the site of the movement and indicate when it had spread to the eyelids. Following each attack the right arm was more flaccid and its tendon reflexes less active than before. On each occasion the attack was induced on attempting to speak. 
On the fourth day the attacks appeared as on the previous day, both on trying to speak and sometimes when no attempt was made to do so. On each occasion, after a rest of a few minutes, the patient was able to speak freely. There was a "feeling of pulsation " in the right hand and a sensation of "pins and needles" both there and in the right foot. Sympathetic induction of emotion was still very active, for the patient was deeply distressed and felt pain acutely when an operation case was returned to the ward. The tendency to this sympathetic emotion decreased very much during the week following the operation and after that was less easily aroused.

The difficulty with speech was at its maximum on the fifth day. Up to that time the periods of aphasia had steadily become more frequent and longer until they persisted for as long as ten minutes at a time. They occurred chiefly in association with the local twitching movements of the face and eyelids. The patient explained that after he had said a few words he felt " as if the brain got tired; he finally got to the stage when he got only part of a word out and then no words would come."

On the seventh day the local "fits" which had been present for four days no longer appeared, and the difficulty with speech was no worse than it had been before the operation.

On the twentieth day there was still slight impairment of cottonwool sensation over the right arm, but the appreciation of pain was as good as before over the left arm. Free voluntary movements of the fingers and toes were possible on awakening from sleep but soon tired. Over the leg there was a slight difference in cottonwool sensation between the right and left legs; and the same difference was apparent for pain and thermal sensations. Changes of position were appreciated in movements of the right ankle but not in those of the toes. Vibration sense had improved in the right leg but was still less easily appreciated than in the left leg. There was slight impairment of deep pressure sense in the right tendo Achillis. Movements at the right hip were good, at the knee they were possible in all directions but weak, while at the ankle and toes there were limited movements of flexion and extension. Movements of the toes appeared for the first time since the onset of the illness. Five weeks after the operation speech was normal, movements of the right arm, ankle and toes could be performed much more freely and for longer periods, and the appreciation of sensation of all types was as good on the right side as on the left.

Spontaneous "Stereognostic" Sensations.-As soon as the patient became conscious on recovering from the anæsthetic he thought he was holding an object in the palm of the right hand. So real was the impression that he felt with the left hand to find if there was actually anything there. During the first two days, this sensation was constantly present and very definite. The shape and size of the objects felt and the objects the patient recognised varied from time to time. At one time he felt a smooth, round object which he described as "like a ball which just fits into the palm of my hand." Again, he felt " something rough and jagged and hard like a piece of road granite." Later he felt " a flat round object-like a ladies' small mirror." He also felt " a long, round object like a long, round pencil case," and an object "like a matchbox." Not only did he appreciate the size, shape and consistency of the object but he was able either to recognise it or to compare it with an object well known to him.

On the third and fourth days these sensations were still present and very real to the patient but appeared at gradually increasing intervals. They were, however, not so obvious as on the two days immediately after the operation. At this stage, he discovered accidentally that, when a thermometer was placed in the palm of the left hand, he could also feel an object of the same size and shape which he recognised as " another thermometer" in the palm of the right hand. When an object such as a matchbox, a penny or a fountain pen was placed in the palm of the left hand he felt it perfectly well there and was able to recognise it, but at the same time he also felt a fainter sensation of the presence of another object exactly the same in the palm of the right hand. When a silk handker. 
chief was placed in the left hand he had the same sensation of the presence of a soft silky material in the right hand, though less pronounced than in the left hand. A further test suggested that thermal sensation was also involved in this phenomenon. When a cold penny was placed in the palm of the left hand there was at once a transient feeling of coldness in the right hand as if a cold object had been placed therein. He volunteered the statement that this sensation of coldness in the right hand persisted only as long as did the feeling of coldness in the left hand on first feeling the penny. He could appreciate gross variations in size and shape when an object was placed in the right hand but failed to appreciate the finer variations. He could recognise the general shape and size of a watch but failed to recognise its handle. When an object such as a fountain pen was placed in the right hand he recognised its general size and shape but could not identify it ; when it was withdrawn gently the sensation of its presence there persisted for at least one minute afterwards. With successive tests this persistence of sensation became weaker and weaker and finally disappeared.

The spontaneous sensations of objects in the right hand were less frequent and fainter on the fifth and sixth days, and on the seventh day had entirely disappeared. The sensation of the presence of an object in the right hand when one was placed in the left hand had also disappeared at the same time. In addition, as mentioned above, the speech defect had reverted to the pre-operation condition, the local fits in the right side of the face, eyelids and foot no longer appeared, and the "pins and needles" in the hand and foot had disappeared.

On the ninth day there was a slight return of the feeling of the presence of a body in the right hand. The patient felt that the right hand was full, waited for some time to see if he could recognise the object therein, failed to do so, moved the left hand across, straightened out the fingers and then the sensation at once disappeared.

There was a further return of the phenomenon on the twelfth day. The patient had been smoking a cigarctte and holding it between the first and second fingers of the left hand. About fifteen minutes after he had finished and discarded it, he felt that he was holding a cigarette between the first and second fingers of the right hand. The sensation was quite as definite as that produced when he was actually holding the cigarette between the fingers of the left hand. He felt that there was something between the two fingers of the right hand and at once recognised it as a cigarette. He then felt with the left hand between the first and second fingers of the right hand, separated and rubbed them and the sensation then disappeared. On the twenty-first day the patient felt, on placing a cigarette in his mouth, a box of matches in the right hand. On the twenty-fourth day indefinite sensations of something in the right hand were constantly present. From the twenty-sixth day to the thirty-second day the sensations were very definite and the patient felt objects such as boxes and coins in the right hand. After that the sensations became indefinite and faded away.

\section{DISGUSSION.}

The most interesting features of the case described were those concerned witn the appreciation of size, shape and consistency. These exhibited three different forms, viz. :-

(1) Spontaneous "stereognostic" sensations in the right hand, most pronounced immediately after the removal of the tumour, gradually decreasing during the following six days, disappearing on the seventh day, but reappearing at infrequent intervals for five weeks after the operation.

(2) A feeling that objects were present in the right hand when they were actually present and felt in the left hand, a sensation which was at its maximum on the fourth day after the operation. 
(3) Persistence of sensation in the right hand after an object had been removed from it.

In order to understand the circumstances under which these sensations appeared it is necessary to review the condition and habits of the patient from the onset of his illness. For fifteen months at least a tumour had been present impinging upon the cerebral cortex immediately behind the fissure of Rolando close to the middle line, displacing this portion of the cortex and adjacent brain tissue below and also at a later stage displacing the neighbouring tissues in front of, lateral to and behind it. Definite symptoms of interference with both the sensory and motor areas of the cerebral cortex for the leg had been present since the onset, and had gradually increased in such a way as to suggest that the greater part of both areas had become more or less involved. Though, during this time, the patient was still able, save for a short period after each attack, to grasp large objects with the right hand and to use it for purposes which did not require the appreciation of the finer degrees of sensation for their performance, the increasing motor disability in the right arm and hand had compelled him to rely more and more upon the left hand for the handling of small objects. This gradual education of the left hand in actions usually performed by the right hand had proceeded for nearly fifteen months. In addition there was some evidence of disturbance in the optic thalamus and neighbouring structures on the left side in the form of pain deep in the right arm, emotional disturbances and tremor in the right hand and arm on movement. Such disturbances were probably due as much to interference with the circulation as to anything else, as were also the symptoms of interference with those portions of the motor and sensory cortex previously mentioned. At the operation a tumour was shelled out from a cavity formed by the displacement of the pre- and post-rolandic areas of the cerebral cortex close to the middle line. With the removal of the tumour the cavity filled with blood clot and the circulation in the compressed portion of the cerebral tissues and those adjacent to them was probably much disturbed. Because of the replacement of the tumour by blood clot and the extreme degree of displacement of the parts immediately adjoining the cavity, those parts which were at some distance from it were probably affected to a greater extent by changes in the circulation than were those immediately adjacent to it. That this was probably true was supported by the occurrence of speech defects, tingling in the right hand and motor Jacksonian fits confined to the right side of the face and the eyelids, all reaching their maximum on the fifth day after the operation and then gradually decreasing until the seventh day, when they no longer appeared. That something was also happening but in a lesser degree to the motor and sensory areas for the leg close to the middle line was suggested by the appearance of tingling and "twitching" movements in the right foot on the second and third days after the operation. That there were also variations in the deeper-seated structures was suggested by the rapid diminution in sympathetic emotion, a feature of the illness which had caused the patient much distress before the 
operation. It was probable that, following the removal of the tumour, there was an increase in the circulation of the parts surrounding the cavity, reaching its maximum about the fifth day and then gradually returning to normal; whereas the circulation of those parts immediately adjacent to the cavity was readjusted in a much more gradual manner. Such then was probably the pathological background upon which the sensations to be described were based.

The first variation in sensation to be discussed is that which for convenience has been termed spontaneous "stereognostic" sensation. The details of the phenomenon have been described under the clinical features of the case and it is necessary only to mention that the patient had at different times the sensation of the presence of bodies of different sizes, shapes and consistencies in the palm of the right hand ; that he sometimes merely compared the objects thus felt with objects well known to him, but more often actually recognised them as such without any hesitation; and that these sensations were present constantly for the first two days after the operation and appeared at increasing intervals and less obviously thereafter. Objects such as "a smooth, round ball," "something rough and jagged and hard like a piece of road granite," " a flat, round object like a ladies' small mirror," " a long, round object like a long, round pencil case," a matchbox and a cigarette were felt at different times. At first the sensation of the presence of an object in the right hand was so real to the patient that he had to feel with the left hand to be sure there was nothing there. Subsequently, however, there was some difficulty in recognising the object though there was no doubt in the patient's mind that there was something there. On one occasion the sensation of the presence of a cigarette in the right hand appeared fifteen minutes after the patient had been holding a cigarette in the same position in the left hand. On another occasion, three weeks after the operation, the patient wanted a box of matches, felt that he was holding it in the right hand, but on feeling for it with the left hand found that it was not there but lying on the bed.

Some clue to the features associated with the phenomenon was supplied by the observation that on one occasion passive movement of the fingers of the right hand caused the sensation to disappear, while on a second occasion passive movement of the fingers and rubbing them caused it to disappear. Thus it was suggested that the position in which the fingers of the right hand chanced to be had something to do with the feeling that an object was present therein. This suggestion was supported by the fact that after the gentle removal of an object from the right hand, the sensation of its presence there persisted until a movement of the fingers dispelled it. Unfortunately, after this aspect of the question had been appreciated, there arose no further opportunity of deciding whether the position of the hand and fingers at the time corresponded with that in which they would have to be to grasp the object which the patient felt was present. It was, however, noticed by the patient that in each case the hand and fingers were in the position they would have to be 
to hold the object felt, with one exception that when feeling a ball the hand was in a position different from that which it usually assumed when the patient was holding a ball.

It is probable, however, that there were other factors concerned in the production of the spontaneous sensations. It was known that there was some disturbance, probably circulatory in nature, involving the parts concerned with the motor aspect of speech, the motor areas for the facial movements and the movements of the eyelids, and the sensory area for the hand for at least one week after the operation; and that this disturbance revealed itself in the form of " motor" aphasia, motor Jacksonian fits confined to the right side of the face and the upper eyelids, and tingling in the right hand. It is reasonable to suppose therefore that this disturbance played some part in the production of the spontaneous "stereognostic" sensations experienced by the patient. Sensations of a similar type occur in association with local lesions in other parts of the brain and are usually definitely. related to the function of the part concerned. The sensations of smell occurring in connection with lesions of the uncinate gyrus are of this type, and vary in different subjects in their resemblance to smells previously experienced. Such easily recognised smells as those of lysol and of chloroform have been experienced by patients observed by the writer. Perhaps lesions of the optic radiations and the visual centres provide a more useful analogy. For example, lesions of the visual centres may be associated with " unformed" sensations such as flashes of light and colour while those involving the optic radiations further forward result more frequently in visual hallucinations which take the shape of "formed" objects, such as figures in fancy dress, as in one case observed by the writer. It is possible that this analogy will explain the occurrence of spontaneous sensations involving the association of tactile sensations, sensations of deep pressure and the sense of position in the case under discussion. It is known that motor or sensory Jacksonian fits occur as the result of a local lesion at or near the particular part of the cortex concerned in those who are predisposed to them. Such fits, when of the sensory type, usually take the form of simple sensations such as tingling or " pins and needles," and are thus analogous to the flashes of light and colour which occur sometimes with lesions at or near to the visual cortex. Of this type of fit the earlier history of the case under discussion provided numerous examples. It is suggested that a lesion at or near the surface irritates or releases the functions of widely divergent sensory structures in such a way that they can be interpreted by the higher centres as only "unformed" sensations. If lesions of the suprathalamic sensory structures at a lower level be regarded as analogous with those of the optic radiations far forward, it might be expected that a lesion there, such as might be produced by comparatively widespread circulatory changes, would produce a definite spontaneous "formed" sensation which would result in the recognition by the patient of an object of the same size, shape and consistency as one which he had felt previously. The explanation suggested is that a disturbance affecting the sensory structures 
at or near the surface initiates sensory impulses which are so scattered and relatively infrequent that when associated at a higher level they can be recognised as only the simpler forms of sensation, whereas one affecting the sensory structures at a deeper level, where they are more closely arranged, initiates sensory impulses which are so numerous and varied that when associated they can be interpreted by the higher centres as due to objects which have the properties of size, shape and hardness or softness. It seems reasonable to suppose that such a temporary disturbance probably occurred in the case reported, and initiated by " irritation " a flood of sensory impulses to the higher centres, where they were interpreted as due to the presence of an object of which the patient had had previous knowledge in the hand from which the fibres irritated normally conveyed sensory impulses.

In connection with the optic radiations a similar phenomenon in the form of a periodical visual hallucination was observed by Pavlov in a dog in which a portion of the "cortical cutaneous analyser" had been extirpated. It was suggested that this was "probably due to the distortion of the effect upon the cortex of the external visual stimuli by local, internal stimuli originating in the extending scar," and that "many similar cases of illusions in man are probably due to the interference of similar cortical stimuli of internal local origin." It might be asked why lesions of the suprathalamic sensory structures do not result more often in spontaneous "stereognostic" sensations. It is possible that in many cases they are overlooked because the patient does not regard them of any importance, and in most cases that the lesion is so extensive and severe that the transmission of sensory impulses is impossible. The conditions necessary for the occurrence of such sensations would appear to be the presence of a lesion involving only a portion of the sensory structures and leaving the remainder only slightly or not at all affected.

There is yet another possible explanation of the curious sensory phenomena which occurred in this case, viz., the effect that a local lesion such as a tumour, followed by the effects of its removal, would have upon all the other cerebral functions, including those which are usually grouped under the heading of psychical processes. It was evident that following the operation a serious degree of mental fatigue was present, as was shown by the manner in which the speech defects arose ; and it is possible that this might have been responsible for the confusion and misinterpretation of sensations. The most obvious form of confusion was the feeling that an object was present in the right hand when one was placed in the left hand, while at the same time its presence in the left hand was fully appreciated by the patient. The placing of a thermometer, a fountain pen, a matchbox, a penny or a cigarette in the left hand produced in every case the feeling that a similar object was present in the same position in the right hand. In addition, a silk handkerchief was felt in the right hand as well as in the left, and the sensation of coldness produced by placing a cold penny in the left hand was appreciated in the right hand as well. That some higher cerebral process was probably involved was suggested by an 
experience which the patient had as late as three weeks after the operation, when on placing a cigarette in his mouth he immediately felt the necessary box of matches in the right hand. The "crossing of sensations" occurred only during the first week after the operation. The normal sensory impulses from the left hand were correctly interpreted as being due to an object in the left hand, and it is possible that the abnormal impulses arising at a higher level were at the same time interpreted as being due to a similar object in the right hand.

In this connection an observation already mentioned is of some interestthe fact that the sensation of the presence of a " phantom " cigarette in the right hand was appreciated fifteen minutes after one had been held in a similar position in the left hand. On this occasion there was, of course, no sensation of the presence of a cigarette in the left hand at the same time. It is possible that during the education of the left hand in the handling of objects usually handled by the right hand, a similar "lead" had been given to the interpreta. tion of the impulses arising at a higher level on the opposite side as a result of the changes following the operation, quite apart from the experience in handling objects with the right hand before the onset of the illness. It was significant that the objects felt were confined to those which the patient had at some time held in his left hand during his illness. That fatigue was at least a factor in the development of the sensations was suggested by the fact that they did not as a rule occur after a period of sleep and they decreased in intensity as mental fatigability lessened during convalescence.

These suggestions as to the cause of the sensory phenomena described are merely put forward tentatively. It is possible that all three of the factors mentioned played some part in the production of the sensations experienced by the patient.

\section{SUMMARY AND GONGLUSIONS.}

1. The history, clinical investigation, operative treatment and postoperative symptoms of a case of meningioma of the postrolandic sensory cerebral cortex are described in detail.

2. Following the operation at which the tumour was removed three types of abnormal "stereognostic" phenomena occurred, viz. :-

(a) Spontaneous sensations of the presence of solid objects in the affected hand ;

(b) Sensations of the presence of solid objects in the affected hand when they were actually present and felt in the unaffected hand ;

(c) Persistence of the sensations of the presence of objects in the affected hand for an appreciable interval after they had been removed therefrom.

3. The probable causes of these sensory phenomena are discussed, viz :-

(a) The effect that accidental positions of the affected hand might have in the production of spontaneous "stereognostic" sensations ; 
(b) The effect that local impulses, arising in the sensory structures immediately above the thalamus as a result of post-operative circulatory changes in that region, might have in the production of spontaneous "formed" sensations, in contrast with the effect of lesions of or close to the cerebral cortex in the formation of spontaneous " unformed" sensations;

(c) The effect that post-operative mental fatigue might have in the misinterpretation of the local sensory impulses arising at the suprathalamic level.

4. It is possible that the local sensory impulses arising as the result of post-operative circulatory changes immediately above the thalamus were the main factor and that mental fatigue and accidental positions of the affected hand were contributory factors in the production of the sensory phenomena described.

The writer wishes to thank Dr. Neill Hobhouse, Mr. Cecil P. G. Wakeley and Dr. Carnegie Dickson for permission to publish the notes of this case. 